\title{
Effectiveness of Rituximab for the Treatment of Rheumatoid Arthritis in Patients with Prior Exposure to Anti-TNF: Results from the CORRONA Registry
}

\author{
Leslie R. Harrold, George W. Reed, Ashwini Shewade, Robert Magner, Katherine C. Saunders, \\ Ani John, Joel M. Kremer, and Jeffrey D. Greenberg
}

\begin{abstract}
Objective. To characterize the real-world effectiveness of rituximab (RTX) in patients with rheumatoid arthritis.

Methods. Clinical effectiveness at 12 months was assessed in patients who were prescribed RTX based on the Clinical Disease Activity Index (CDAI). Change in CDAI was calculated (CDAI at 12 mos minus at initiation). Achievement of remission or low disease activity (LDA; CDAI $\leq 10)$ among those with moderate/high disease activity at the time of RTX initiation was compared based on prior anti-tumor necrosis factor agent (anti-TNF) use ( $1 \mathrm{vs} \geq 2)$ using logistic regression models.

Results. Patients $(\mathrm{n}=265)$ were followed for 12 months with a mean change in CDAI of -8.1 (95\% CI -9.8--6.4). Of the 218 patients with moderate/high disease activity at baseline, patients with 1 prior anti-TNF (baseline CDAI 25.0) demonstrated a mean change in CDAI of -10.1 (95\% CI $-13.2--7.0$ ); patients with $\geq 2$ prior anti-TNF (baseline CDAI 30.0) demonstrated a mean change of -10.5 (95\% CI $-12.9--8.0)$. The unadjusted OR for achieving LDA/remission in patients with moderate/high disease activity at baseline exposed to $\geq 2$ versus 1 prior anti-TNF was $0.40(95 \% \mathrm{CI}$ 0.22-0.73), which was robust to 4 different adjusted models (OR range 0.38-0.44).

Conclusion. A good clinical response was observed in all patients; however, patients previously treated with 1 anti-TNF, who had lower baseline CDAI and a greater opportunity for clinical improvement compared with patients previously treated with $\geq 2$ anti-TNF, were more likely to achieve LDA/remission. (First Release May 1 2015; J Rheumatol 2015;42:1090-8; doi:10.3899/jrheum.141043)
\end{abstract}

Key Indexing Terms:

RHEUMATOID ARTHRITIS RITUXIMAB ANTIRHEUMATIC AGENTS REGISTRIES

Rheumatoid arthritis (RA) is a chronic, systemic autoimmune disease characterized by inflammation of the joints. An

From the University of Massachusetts Medical School, Worcester; Corrona LLC, Southborough, Massachusetts; Genentech Inc., South San Francisco, California; Albany Medical College and The Center for Rheumatology, Albany; New York University (NYU) School of Medicine, New York, New York, USA.

The CORRONA Rheumatoid Arthritis registry has been supported through contracted subscriptions in the last 2 years by Abbvie, Amgen, AstraZeneca, Genentech, Horizon Pharma, Eli Lilly, Novartis, Pfizer, Vertex, and UCB. Support for third-party writing assistance for this manuscript, furnished by Vivian Chen, PharmD, and Eric Deutsch, PhD of Health Interactions, was provided by F. Hoffmann-La Roche Ltd.

L.R. Harrold, MD, MPH, Department of Medicine, University of Massachusetts Medical School; G.W. Reed, PhD, Department of Medicine, University of Massachusetts Medical School, and Corrona LLC; A. Shewade, MS, Genentech Inc.; R. Magner, MPH, Department of Medicine, University of Massachusetts Medical School; K.C. Saunders, MS, Corrona LLC; A. John, BSN, MPH, Genentech Inc.; J.M. Kremer, $M D$, Department of Rheumatology, Albany Medical College, and The Center for Rheumatology; J.D. Greenberg, MD, MPH, Department of Medicine, Division of Rheumatology, NYU School of Medicine.

Address correspondence to Associate Professor L.R. Harrold, University of Massachusetts Medical School, 55 Lake Ave. North, Worcester,

Massachusetts 01655,USA.E-mail: Leslie.Harrold@umassmed.edu

Full Release Article. For details see Reprints/Permissions at jrheum.com Accepted for publication March 10, 2015. improved understanding of the immunologic processes associated with RA in the past decade has led to advances in disease management, including approval of biologic therapies that target the various autoimmune molecules involved in the pathophysiology of RA. For patients who fail to respond to nonbiologic disease-modifying antirheumatic drug (DMARD) therapy or who develop an inadequate response over time, anti-tumor necrosis factor (anti-TNF) therapies are typically the first-choice biologic. Anti-TNF have demonstrated the ability to reduce signs and symptoms of RA, inhibiting the underlying radiographic progression, improving physical function, and reducing disability $1,2,3,4,5$.

However, the effectiveness of anti-TNF varies among patients, likely attributable to the differences in the activity of pathophysiological pathways and cytokines that mediate the RA disease process. Whether it is attributable to a primary or secondary treatment failure, as many as $60 \%$ of patients may have an inadequate response to anti-TNF therapy ${ }^{6}$. Switching to another biologic therapy with an alternate mechanism of action, such as rituximab (RTX), may be an effective strategy in anti-TNF-refractory patients. RTX is a chimeric murine/human monoclonal antibody that binds specifically to the antigen CD20. In the United States, RTX

Personal non-commercial use only. The Journal of Rheumatology Copyright $\odot$ 2015. All rights reserved 
is indicated in combination with methotrexate (MTX) for the treatment of patients with active disease who have not responded to treatment with $>1$ anti-TNF.

While RTX has been shown to be effective in randomized controlled trials (RCT) among patients with RA who have shown an inadequate response to anti- $\mathrm{TNF}^{7,8,9,10}$, these study populations may not accurately reflect real-world patients receiving RTX because of the strict inclusion and exclusion criteria of these trials ${ }^{11,12}$. In clinical practice, many patients initiating RTX have lower disease activity levels compared with those enrolled in anti-TNF RCT, a factor that further influences evaluations of effectiveness ${ }^{12,13,14}$. Most observational data examining the effectiveness of RTX in routine clinical practice have been reported from European registries ${ }^{15,16,17,18,19,20,21,22}$, with scant information currently available from the United States ${ }^{23}$. There is tremendous value in examining US cohorts of patients with RA to assess effectiveness because of the differences in treatment practices and patient characteristics. In many European countries, access to biologic agents is restricted based on patient disease activity and dosing limits. In contrast, medication selection and dosing in the United States are typically based on the rheumatologist's assessment of treatment failure or success ${ }^{12,24}$. Additionally, patients in the United States are heavier than those in Europe ${ }^{25}$; thus, given the current label for a fixed dose, US patients may receive fewer $\mathrm{mg} / \mathrm{kg}$ of medication per infusion.

Given the lack of data on outcomes with usual care in US-based cohorts, the objective of our study was to characterize the real-world use and effectiveness of RTX in a large cohort of patients enrolled in the CORRONA (COnsortium of Rheumatology Researchers Of North America) registry. In particular, we sought to examine the effectiveness of RTX to reduce disease activity and achieve low disease activity (LDA) or remission.

\section{MATERIALS AND METHODS}

Data source. CORRONA is an independent, prospective, observational registry of patients with arthritis who were enrolled by participating rheumatologists at both academic and private practice sites ${ }^{26,27}$ with data gathered from both patients and their treating rheumatologists. As of March 3, 2014, data have been collected from $>39,956$ patients and $>600$ participating rheumatologists, yielding $>111,303$ patient-years of followup. Approvals for data collection and analyses for academic and private practice sites were obtained from local and central institutional review boards, respectively.

Study population. Between February 28, 2006, and December 5, 2011, 30,274 patients with RA were enrolled in the CORRONA registry, of which 615 patients were newly prescribed with RTX after prior exposure to $>1$ anti-TNF in accordance with the RTX label indication. No disease activity or comorbidity exclusion criteria were required for patients with RA enrolled into the consortium registry. Details on patient selection are presented in Appendix 1.

Measures and data collection. Data from the CORRONA registry were analyzed to assess RTX treatment outcomes in usual care settings. Data were collected from both patients and their treating rheumatologists using questionnaires that gathered information on disease severity and activity (including components of the American College of Rheumatology response criteria), comorbidities, use of medications including DMARD, and adverse events ${ }^{26,27}$. Only reported first-time users of RTX were considered; patients who restarted the drug were excluded. Baseline data were obtained at the time of RTX initiation. In cases where RTX initiation occurred between visits, the prior visit was considered the baseline. Followup assessments were requested at 4-month intervals for the registry and completed during routine clinical encounters.

Use of nonbiologic and biologic DMARD was recorded at the time of the routine clinical visit. Data elements also documented at the time of the clinical encounter that were relevant to the current analysis included the Clinical Disease Activity Index (CDAI; 0-76.0) and the physician and patient global assessments of disease activity (each a visual analog scale, $0-10.0 \mathrm{~cm}$ ). Disease remission was defined as CDAI $\leq 2.8$. LDA was defined as a CDAI $>2.8$ and $\leq 10$. Moderate disease activity was defined as CDAI $>10$ and $\leq 22$. High disease activity was defined as CDAI $>22$.

Outcomes. Effectiveness of RTX was examined in patients based on change in CDAI over 12 months. For all patients, we evaluated achievement of remission at 12 months. For those with moderate or high disease activity at the time of initiation, we examined achievement of LDA. Patients who switched from RTX to another biologic were included in the analyses; we used the last observation prior to the switch for evaluation of response. Additionally, safety event rates in terms of all cardiovascular events, serious infections, and malignancies were calculated over the 12-month period.

Statistical analysis. Patient clinical and demographic characteristics were compared between RTX initiators who were eligible for the effectiveness evaluation $(n=265)$ and those who were not $(n=111)$ using standard parametric techniques to assess comparability. In the effectiveness cohort $(n=265)$, patients who were retreated with RTX or switched to other biologic agents over the 12-month period were identified.

To evaluate the effectiveness of RTX, descriptive statistics were used to characterize and compare patients overall and based on prior anti-TNF exposure ( $1 \mathrm{vs} \geq 2$ prior agents). Change in CDAI at 12 months from initiation was calculated. Additionally, unadjusted OR comparing remission or LDA response rates at 12 months of patients with moderate or high disease activity at baseline previously treated with $\geq 2$ vs 1 prior anti-TNF were estimated using multivariable logistic regression.

Adjusted OR were estimated using 4 different approaches that adjusted for baseline variables identified a priori based on their association with the outcome and whether there was a significant univariate difference between patients previously treated with $\geq 2$ versus 1 prior anti-TNF. The variables included a priori as covariates were based on a review of the literature and clinical experience with regard to factors likely to influence response, and included age, sex, duration of RA, CDAI, modified Health Assessment Questionnaire (mHAQ) score, seropositivity [rheumatoid factor (RF) or anticyclic citrullinated peptide antibody (anti-CCP) if RF was not available], number of prior nonbiologics, RTX treatment with no concomitant DMARD, and prednisone use. Propensity scores were derived by modeling exposure to 1 or $\geq 2$ prior anti-TNF as a function of age, duration of RA, CDAI, and mHAQ. Model 1 used population-averaged (PA) logistic regression and excluded patients with propensity scores above the maximum among those with 1 prior anti-TNF or below the minimum among those with $\geq 2$ prior anti-TNF. Model 2 used multivariable logistic regression. Model 3 used PA logistic regression after adjusting for clustering by practice site. Model 4 used PA logistic regression, excluded patients with high and low propensity scores, and included the number of prior anti-TNF exposures ( $\geq 2$ vs 1 ) and propensity score as predictors. For the first 3 models, we adjusted for all the a priori covariates because we used either the full population or the trimmed population. For the fourth model, we adjusted using the propensity score rather than a series of covariates.

The overall safety event rates in the effectiveness population were calculated based on the number of events divided by the duration of exposure in patient-years (PY/100) over the 12-month period. Safety events that were reported by providers were examined. Infections included in the analysis were cellulitis, sinusitis, diverticulitis, sepsis, pneumonia, bronchitis,

Personal non-commercial use only. The Journal of Rheumatology Copyright $\subset$ 2015. All rights reserved. 
gastroenteritis, meningitis, encephalitis, urinary tract infection, upper respiratory tract infection, tuberculosis, joint infection, bursal infection, and all other infections. Cardiovascular events included cardiac arrest, congestive heart failure, myocardial infarction, coronary artery disease, unstable angina, ventricular arrhythmia, cardiac revascularization, stroke, transient ischemic attack, and deep vein thrombosis. Cancer events included breast cancer, lung cancer, lymphoma, skin cancer (not specified, squamous cell, melanoma), and other cancer diagnoses.

\section{RESULTS}

Demographic and clinical characteristics at baseline. By December 5, 2011, there were 265 patients with prior anti-TNF experience who were eligible for the evaluation of medication effectiveness, and were similar in baseline demographics, disease characteristics, and treatment history to those with followup at 12 months $(\mathrm{n}=111)$ but who did not meet eligibility criteria (Table 1). From among the 265 patients who were eligible for the effectiveness evaluation, $44(17 \%)$ switched to another biologic and $221(83 \%)$ remained with RTX. A total of 178 patients (67\%) were retreated with RTX (Appendix 2).

The majority of the 265 patients were women and white, with a median age of 57 years (Table 2). The most common non-RA comorbidity was hypertension, observed in about $35 \%$ of patients. In addition, there was a substantial number of patients with a history of liver disease (9\%), peptic ulcer disease $(9 \%)$, and cancer (15\%). At baseline, patients had a median disease duration of 13 years and significant disease burden, with a median CDAI of 22.2, median swollen joint count of 6 , median tender joint count of 7 , and median mHAQ score of 0.6 .

About $43 \%$ of patients had previous exposure to 1 anti-TNF, and $57 \%$ of patients had previous exposure to $\geq 2$ anti-TNF. Patients previously treated with 1 anti-TNF had better scores in every measurement of disease activity than those previously treated with $\geq 2$ anti-TNF at the time of RTX initiation; however, only median CDAI was significantly different (17.5 vs 24.4, $\mathrm{p}=0.001)$. At the time of RTX initiation, nearly $76 \%$ of patients started RTX in combination with $\geq 1$ nonbiologic DMARD, including 50\% who received concomitant MTX. Patients treated with 1 prior anti-TNF and those treated with $\geq 2$ prior anti-TNF were balanced in terms of concomitant medications. A significantly greater proportion of patients who had received $\geq 2$ prior anti-TNF were treated with concomitant prednisone compared with those who had received 1 prior anti-TNF (49\% vs 33\%, p = 0.008).

Disease activity at baseline and 12 months. At baseline, about $18 \%$ of patients had LDA, $32 \%$ had moderate disease activity, and $50 \%$ had high disease activity (Figure 1). At 12 months, about $11 \%$ of patients had achieved remission, $33 \%$ had LDA, 28\% had moderate disease activity, and $28 \%$ had high disease activity. Overall, the mean CDAI at baseline was 24.2 and decreased to 16.1 at the 12-month followup $\left(\triangle \mathrm{CDAI}_{\text {mean }}-8.1,95 \% \mathrm{CI}-9.8--6.4\right)$. Patients with more severe disease activity at baseline demonstrated a greater

Table 1. Baseline demographics, clinical characteristics, and treatment history of all RTX initiators. Values are n (\%) or median (IQR) unless otherwise specified.

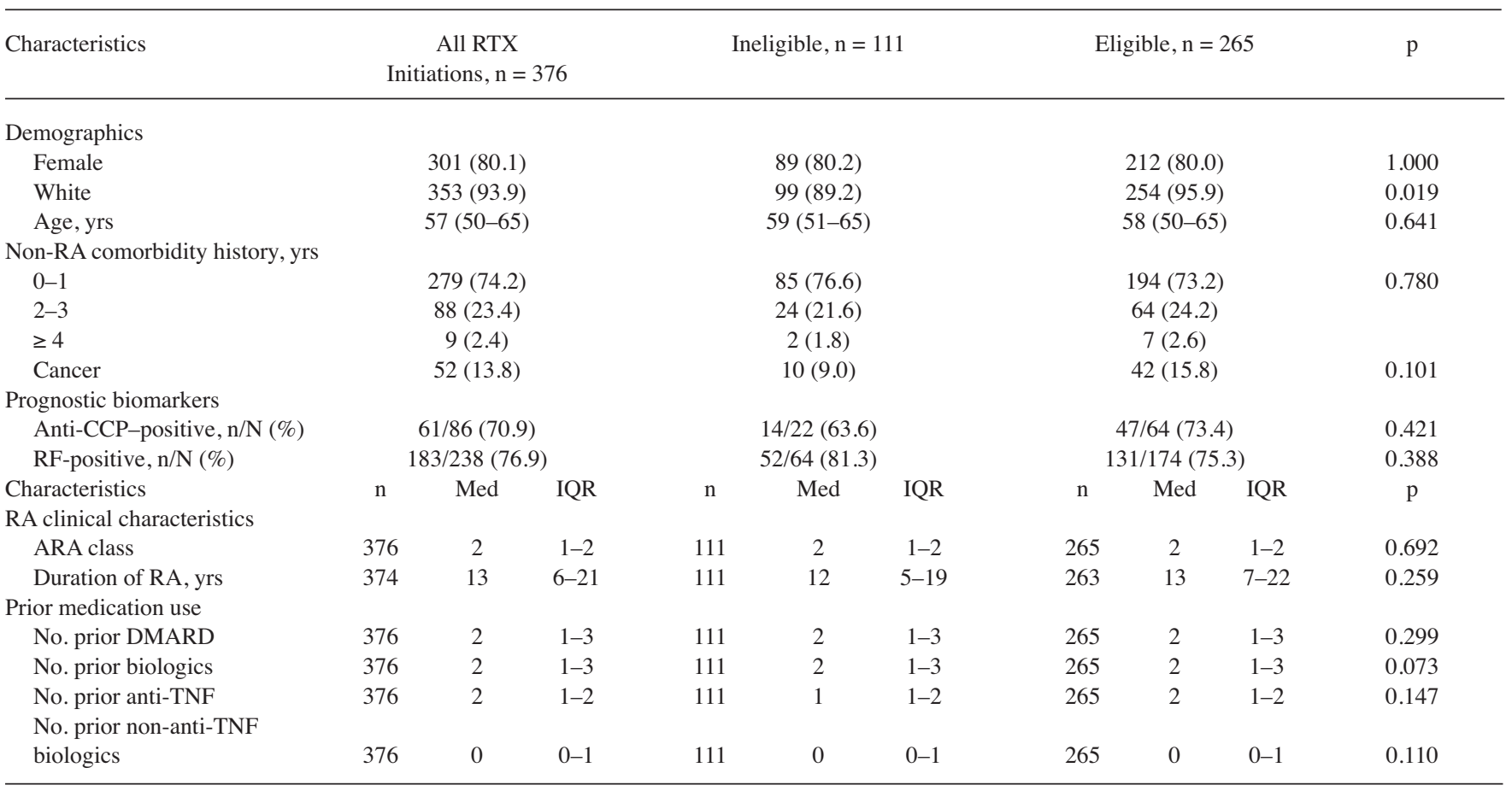

RTX: rituximab; IQR: interquartile range; RA: rheumatoid arthritis; anti-CCP: anticyclic citrullinated peptide antibodies; RF: rheumatoid factor; ARA: American Rheumatism Association; DMARD: disease-modifying antirheumatic drug; anti-TNF: anti-tumor necrosis factor. 
Table 2. Baseline demographics, clinical characteristics, and treatment history of patients with a visit between initiation and 12-month followup. Values are n (\%) or median (IQR) unless otherwise specified.

\begin{tabular}{|c|c|c|c|c|}
\hline Characteristics & $\begin{array}{l}\text { All RTX Initiations, } \\
\qquad \mathrm{n}=265\end{array}$ & $\begin{array}{l}1 \text { Prior Anti-TNF, } \\
n=113\end{array}$ & $\begin{array}{c}\geq 2 \text { Prior Anti-TNF, } \\
n=152\end{array}$ & $\mathrm{p}$ \\
\hline \multicolumn{5}{|l|}{ Demographics } \\
\hline Age, yrs & $57(50-65)$ & $61(52-69)$ & $56(49-63)$ & 0.003 \\
\hline Female & $212(80.0)$ & $91(80.5)$ & $121(79.6)$ & 0.878 \\
\hline White & $254(95.9)$ & $106(93.8)$ & $148(97.4)$ & 0.213 \\
\hline \multicolumn{5}{|l|}{ History of non-RA comorbidities } \\
\hline CV disease & 34 (12.8) & $15(13.3)$ & $19(12.5)$ & 0.855 \\
\hline Hypertension & $94(35.5)$ & $39(34.5)$ & $55(36.2)$ & 0.797 \\
\hline Diabetes & $27(10.2)$ & $10(8.9)$ & $17(11.2)$ & 0.682 \\
\hline Liver disease & $25(9.4)$ & $10(8.9)$ & $15(9.9)$ & 0.834 \\
\hline Peptic ulcer disease & $25(9.4)$ & $10(8.9)$ & $15(9.9)$ & 0.834 \\
\hline Lung disease & $25(9.4)$ & $10(8.9)$ & $15(9.9)$ & 0.834 \\
\hline All cancer & $40(15.1)$ & $17(15.0)$ & $23(15.1)$ & 1.000 \\
\hline Skin cancer & $10(3.8)$ & $4(3.5)$ & $6(4.0)$ & 1.000 \\
\hline Non-skin cancer & $30(11.3)$ & $13(11.5)$ & $17(11.2)$ & 1.000 \\
\hline \multicolumn{5}{|l|}{ Hospitalized for infection in } \\
\hline last $12 \mathrm{mos}$ & $4(1.5)$ & $2(1.8)$ & $2(1.3)$ & 1.000 \\
\hline \multicolumn{5}{|l|}{ RA clinical characteristics } \\
\hline RA duration, yrs & $13(7-22)^{*}$ & $10(6-23)^{\dagger}$ & $14(8-21)$ & 0.326 \\
\hline $\mathrm{TJC}$ & $7(2-12)$ & $5(1-10)$ & $8(3-14)$ & 0.006 \\
\hline SJC & $6(2-10)$ & $5(1-8)$ & $6(2-11.5)$ & 0.008 \\
\hline Patient pain & $53(29-75)$ & $46(20-72)$ & $60(31-76)$ & 0.020 \\
\hline Patient global & $50(31-70)$ & $45(27-65)$ & $55(39-75)$ & 0.005 \\
\hline Physician global & $37(21-53)$ & $30(20-50)$ & $40(23-58)$ & 0.031 \\
\hline mHAQ disability index & $0.63(0.25-1.13)$ & $0.63(0.25-1.00)$ & $0.75(0.38-1.13)$ & 0.271 \\
\hline CDAI & $22.2(12.5-32.5)$ & $17.5(11.0-28.0)$ & $24.4(14.4-35.4)$ & 0.001 \\
\hline RF-positive, $\mathrm{n} / \mathrm{N}(\%)$ & $130 / 173(75.1)$ & $55 / 72(76.4)$ & 75/101 (74.3) & 0.749 \\
\hline Anti-CCP-positive, n/N (\%) & $48 / 65(73.8)$ & $24 / 35(68.6)$ & $24 / 30(80.0)$ & 0.440 \\
\hline \multicolumn{5}{|l|}{ Concomitant medications } \\
\hline No concomitant medications & $61(23.0)$ & $26(23.0)$ & $35(23.0)$ & 0.696 \\
\hline MTX only & $126(47.6)$ & $58(51.3)$ & $68(44.7)$ & \\
\hline Non-MTX, nonbiologic DMARI & RD 47 (17.7) & $17(15.0)$ & $30(19.7)$ & \\
\hline MTX + nonbiologic DMARD & $17(6.4)$ & $8(7.1)$ & $9(5.9)$ & \\
\hline Prednisone & $112(42.3)$ & $37(32.7)$ & $75(49.3)$ & 0.008 \\
\hline Prednisone dose, $\mathrm{mg}^{\S}$ & & & & 0.187 \\
\hline$<5$ & $19(17.0)$ & $9(24.3)$ & $10(13.3)$ & \\
\hline $5-9$ & $45(40.2)$ & $16(43.2)$ & $29(38.7)$ & \\
\hline$\geq 10$ & $48(42.9)$ & $12(32.4)$ & $36(48.0)$ & \\
\hline
\end{tabular}

$* \mathrm{n}=263 .{ }^{\dagger} \mathrm{n}=111 .{ }^{\S}$ Percentage based on total number of patients in each group taking prednisone. IQR: interquartile range; RTX: rituximab; anti-TNF: anti-tumor necrosis factor; RA: rheumatoid arthritis; CV: cardiovascular; TJC: tender joint count; SJC: swollen joint count; mHAQ: modified Health Assessment Questionnaire; CDAI: Clinical Disease Activity Index; RF: rheumatoid factor; anti-CCP: anticyclic citrullinated peptide antibodies; MTX: methotrexate; DMARD: disease-modifying antirheumatic drug.

response to RTX treatment compared with those with less severe disease activity. Specifically, patients with LDA at baseline demonstrated a mean increase in CDAI of $2.3(95 \%$ CI -0.12-4.71), whereas patients with moderate and high disease activity at baseline demonstrated mean decreases in CDAI of $-2.2(95 \% \mathrm{CI}-4.47-0.16)$ and $-15.5(95 \% \mathrm{CI}$ $-12.2--8.4)$, respectively. When the overall sample was stratified by previous exposure to anti-TNF, patients treated with 1 prior anti-TNF demonstrated a mean CDAI of 20.7 at baseline and 13.2 at 12 months; patients with $\geq 2$ prior anti-TNF demonstrated a mean CDAI of 26.8 at baseline and 18.3 at 12 months.
Concomitant use of prednisone. Among patients who started RTX with concomitant prednisone, $31 \%$ of patients increased, $43 \%$ of patients decreased, and $26 \%$ had no change in their prednisone dose at 12 months. There was no significant difference in the change in prednisone dose from baseline between patients with previous exposure to 1 anti-TNF and those with previous exposure to $\geq 2$ anti-TNF. Treatment responses in patients with moderate or high disease activity at baseline. About $82 \%$ of patients had moderate or high disease activity at baseline. Of those patients, $8 \%$ achieved remission and $29 \%$ achieved LDA at 12 months. The mean change in CDAI at the 12-month

Personal non-commercial use only. The Journal of Rheumatology Copyright $\subset$ 2015. All rights reserved. 


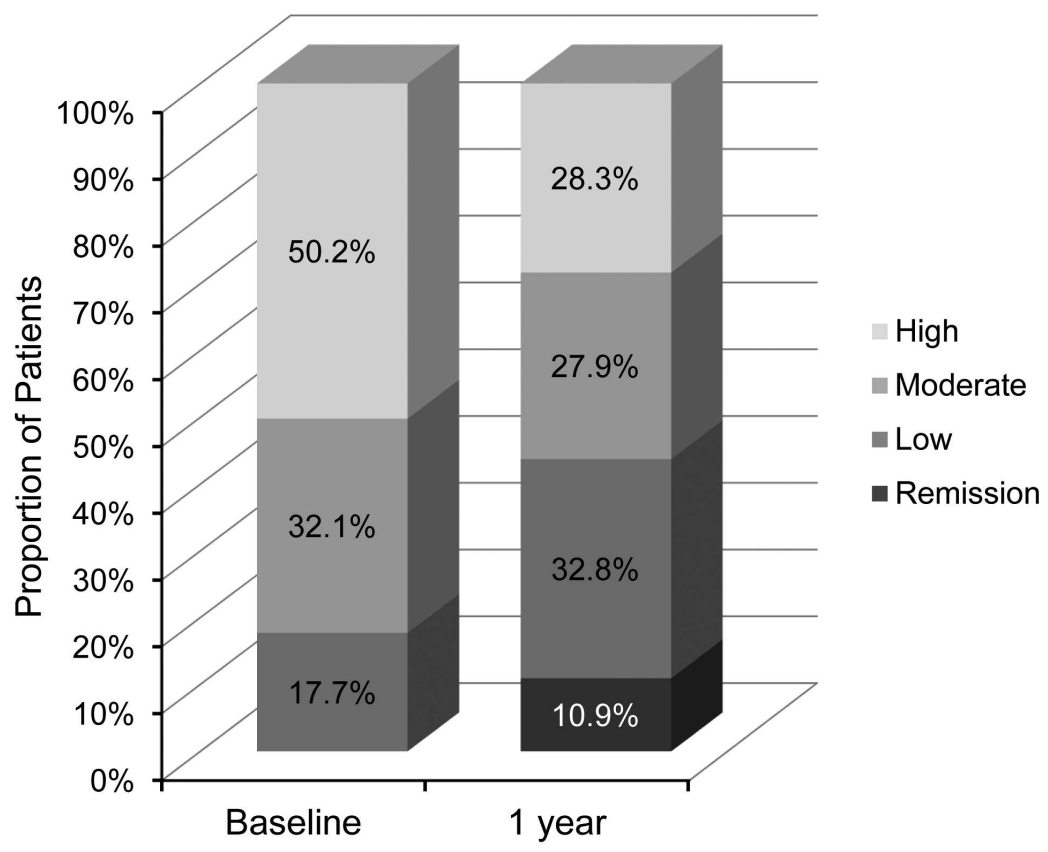

Figure 1. Disease activity at baseline and 12 months $(\mathrm{n}=265)$.

followup in patients with moderate or high disease activity was -10.3 (95\% CI $-12.2--8.4)$. When stratified by previous exposure to anti-TNF, $10 \%$ and $40 \%$ of patients with moderate to high disease activity with previous exposure to 1 anti-TNF achieved remission and LDA at 12 months, respectively (Figure 2). In contrast, $7 \%$ and $22 \%$ of patients with moderate to high disease activity with previous exposure to $\geq 2$ anti-TNF achieved remission and LDA at 12 months, respectively. The mean change in CDAI was similar between patients previously treated with 1 anti-TNF and those previously treated with $\geq 2$ anti-TNF. Patients previously treated with 1 prior anti-TNF demonstrated a mean CDAI of 25.0 at baseline and 14.9 at 12 months $\left(\triangle \mathrm{CDAI}_{\text {mean }}-10.1,95 \% \mathrm{CI}\right.$ $-13.2--7.0)$, whereas patients with $\geq 2$ prior anti-TNF

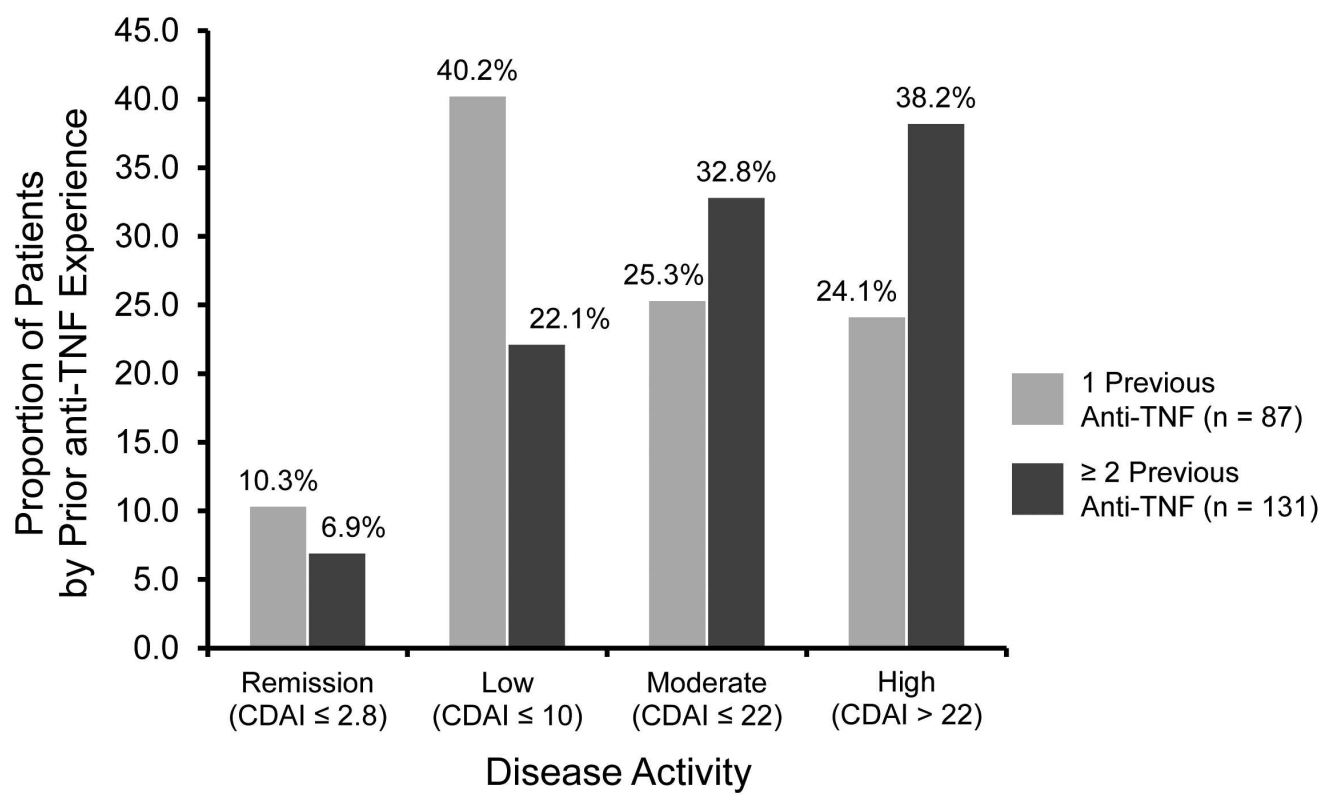

Figure 2. Disease activity at 12 months in patients with moderate/high disease activity at baseline $(\mathrm{n}=218)$ categorized by prior anti-TNF experience. CDAI: Clinical Disease Activity Index; anti-TNF: anti-tumor necrosis factor. 
demonstrated a mean CDAI of 30.0 at baseline and 19.5 at 12 months $\left(\triangle \mathrm{CDAI} \mathrm{I}_{\text {mean }}-10.5,95 \% \mathrm{CI}-12.9--8.0\right)$.

Likelihood of remission or LDA in patients with moderate or high disease activity based on prior anti-TNF exposure. Patients with moderate or high disease activity at baseline who were previously treated with 1 anti-TNF were significantly more likely to achieve remission or LDA at 12 months compared with those previously treated with $\geq 2$ anti-TNF (unadjusted OR $0.40,95 \%$ CI $0.22-0.73$ ). Neither presence of autoantibodies nor cotreatment with a nonbiologic DMARD was associated with improved efficacy in the unadjusted analysis. The adjusted OR for achieving remission or LDA at 12 months in patients with moderate to high disease activity based on prior anti-TNF exposure was further explored using 4 different logistic regression models, including a patient-specific approach, a PA approach, and propensity score-matching approaches (Figure 3). In all 4 models, patients treated with $\geq 2$ prior anti-TNF consistently demonstrated a significantly lower likelihood of achieving remission or LDA compared with patients treated with 1 prior anti-TNF (OR range 0.38-0.44). The addition of seropositivity to the models (RF or anti-CCP if RF was not available) had no effect on the estimates in any of the models.

Safety outcomes. The incidence rate of any cancer was 1.5 events per 100 patient-years (95\% CI 0.6-4.0), while skin, lung, and breast cancer occurred at a rate of $1.1(95 \% \mathrm{CI}$ 0.4-3.5), 0.0 (95\% CI 0.0-1.9), and 0.5 (95\% CI 0.1-3.7) events per 100 person-years, respectively. Ninety-eight infection events were reported, resulting in an overall incidence rate of 36.4 events per 100 person-years (95\% CI 29.8-44.3). Respiratory infections were the most common type observed $(59.2 \%)$, followed by urinary tract infections
$(12.2 \%)$ and cellulitis $(8.2 \%)$. Three serious infection events occurred, resulting in a rate of 1.6 events per 100 person-years (95\% CI 0.5-4.9). Cardiovascular events were infrequent, with 5 events at an incidence of 1.9 events per 100 person-years (95\% CI 0.6-4.0).

\section{DISCUSSION}

In this US-based observational study of patients with longstanding RA cared for by rheumatologists in the usual care setting, RTX appeared effective for the treatment of patients with prior exposure to anti-TNF. Patients treated with $\geq 2$ prior anti-TNF had longer duration of disease and significantly higher disease activity and were more frequently treated with concomitant prednisone compared with those with 1 prior anti-TNF. Despite these differences, improvement in disease activity based on mean change in CDAI at 12 months was similar regardless of the number of prior anti-TNF. When limiting the population to patients with moderate or high disease activity at baseline, those treated with 1 prior anti-TNF consistently demonstrated higher odds of remission or LDA at 12 months compared with patients who received $\geq 2$ prior anti-TNF. This outcome was robust to 4 logistic regression models, including 1 that adjusted for unique differences in drug prescribing and administration by site. These findings are likely related to the lower mean baseline CDAI scores, and thus greater opportunity to achieve LDA or remission among patients treated with 1 prior anti-TNF compared with those treated with $\geq 2$ prior anti-TNF.

At baseline, most patients had moderate or high disease activity and comorbidities not typically observed in RCT, including a history of liver disease, peptic ulcer disease, and

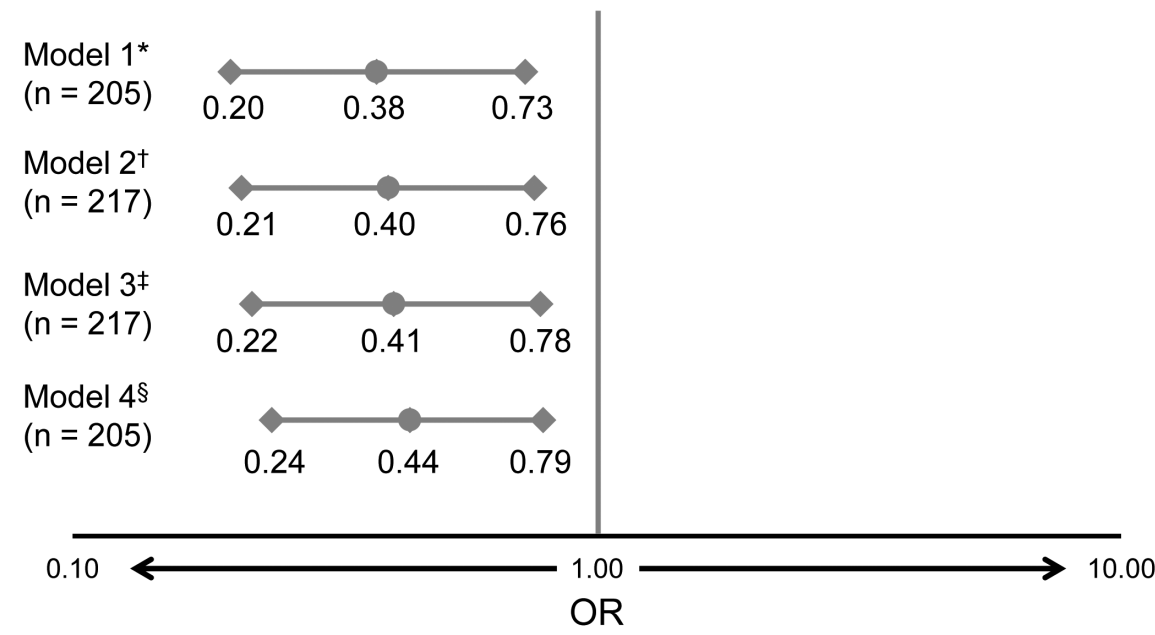

Figure 3. OR ( $\geq 2$ prior anti-TNF vs 1 prior anti-TNF) of achieving remission or LDA at 12 months in patients with moderate/high disease activity at baseline by 4 logistic regression models. * Logistic regression averaged over the population, excluding outliers for propensity scores. ${ }^{\dagger}$ Logistic regression. ${ }^{\ddagger}$ Logistic regression averaged over the population after accounting for differences by practice site. ${ }^{\S}$ Logistic regression averaged over the population, excluding outliers and adjusting for prior TNF use ( $\geq 2$ vs 1 ) and propensity scores matched for quintiles. anti-TNF: anti-tumor necrosis factor; LDA: low disease activity.

Personal non-commercial use only. The Journal of Rheumatology Copyright (C) 2015. All rights reserved. 
cancer $^{4,7,10}$. The demographics and RA clinical characteristics of patients treated with RTX in our study were largely similar to US observational cohorts of patients with RA switching to their second or third anti-TNF ${ }^{24}$, with the exception of worse patient global, patient pain, and physician global assessments. Perhaps, as expected, a larger proportion of patients in our study had moderate or high disease activity (82\%) compared with the cohort treated with anti-TNF $(56-61 \%)^{24}$. This suggests that physicians are preferentially prescribing RTX over anti-TNF to the more active and severe RA cases.

The results of our study are supported by a previously published pooled analysis of data from 10 European registries (Collaborating European REgistries for Rituximab in RA; CERRERA), in which patients starting RTX who were treated with 1 prior anti-TNF demonstrated substantial improvement in disease activity as measured by the 28 -joint Disease Activity Score at 6 months. In fact, the response was greater than that seen in patients treated with $\geq 2$ prior anti-TNF. In multivariate analyses, failure to respond to 1 previous biologic agent was a significantly better predictor of the European League Against Rheumatism good response at 3 and 6 months compared with failure to respond to 2 or more biologic agents (OR 1.89, 95\% CI 1.02-3.51) $)^{15}$. This is similar to what we found regarding a decreased likelihood of achieving remission or LDA. While mean change in CDAI following RTX was similar in patients with 1 versus $\geq 2$ prior anti-TNF, the higher levels of disease activity in those with exposure to $\geq 2$ prior anti-TNF resulted in a lower likelihood of achieving remission or LDA.

The optimal sequence of biologic treatment in patients with an inadequate response to anti-TNF continues to be the subject of much debate. Data from registries have suggested that the response rates of patients switching to a second or third anti-TNF are often lower than those of patients treated with their first anti-TNF ${ }^{28,29}$. Meanwhile, a paradigm shift in treatment strategies has occurred, with a greater focus on "tight control", or achievement of a predefined level of LDA or remission within a reasonable period of time ${ }^{30,31}$. Taking these points into consideration, the results from the current study add to the growing body of evidence seen with other biologics that earlier initiation of RTX may lead to tighter control of disease activity and improved clinical outcomes in patients with an inadequate response to an anti-TNF ${ }^{32}$. A comparative analysis directly comparing RTX to other interventions after failure of previous anti-TNF treatment is necessary to validate these conclusions; such a study is currently being conducted and its results are forthcoming ${ }^{33}$.

There are strengths and limitations to this analysis. Our study is derived from a national cohort of "real-world" patients with detailed clinical information and is one of the first reports of RTX effectiveness in a US patient population. However, the care received by patients reflects current clinical practice in the United States and is merely observational in nature. For example, there was no mandate to retreat with RTX at 4 to 6 months, thus the estimates of effectiveness here may be underestimated. Patients who were treated with $\geq 2$ anti-TNF had a longer disease duration and significantly worse disease activity. They may be less likely to respond to any drug, regardless of its mechanism of action. Additionally, we were unable to assess radiological outcomes, neither were we able to investigate any potential decreases in immunoglobulin levels, a concern with RTX retreatment, in this short-term study. The all-comers design included all RTX initiations regardless of the reason for discontinuation of the previous biologic - a factor that may influence effectiveness. Lastly, these patients were identified from a registry and not a population-based sample; thus, it is unknown how representative they are of the typical RTX-treated patient with RA.

In our study, patients with RA receiving RTX had active and severe RA and a substantial burden of comorbid conditions. Based on the mean change in CDAI, patients with exposure to 1 and $\geq 2$ prior anti-TNF demonstrated good clinical response when treated with RTX. However, patients exposed to 1 prior anti-TNF, who had lower CDAI at baseline and a greater opportunity for clinical improvement, achieved LDA or remission more frequently compared with those exposed to $\geq 2$ prior anti-TNF. This suggests that, consistent with data from studies of other biologics, earlier intervention with RTX in anti-TNF-experienced patients may be preferable.

\section{REFERENCES}

1. Keystone EC, Kavanaugh AF, Sharp JT, Tannenbaum H, Hua Y, Teoh LS, et al. Radiographic, clinical, and functional outcomes of treatment with adalimumab (a human anti-tumor necrosis factor monoclonal antibody) in patients with active rheumatoid arthritis receiving concomitant methotrexate therapy: a randomized, placebo-controlled, 52-week trial. Arthritis Rheum 2004; 50:1400-11.

2. Weinblatt ME, Kremer JM, Bankhurst AD, Bulpitt KJ, Fleischmann RM, Fox RI, et al. A trial of etanercept, a recombinant tumor necrosis factor receptor:Fc fusion protein, in patients with rheumatoid arthritis receiving methotrexate. N Engl J Med 1999;340:253-9.

3. Klareskog L, van der Heijde D, de Jager JP, Gough A, Kalden J, Malaise M, et al; TEMPO (Trial of Etanercept and Methotrexate with Radiographic Patient Outcomes) study investigators. Therapeutic effect of the combination of etanercept and methotrexate compared with each treatment alone in patients with rheumatoid arthritis: double-blind randomised controlled trial. Lancet 2004;363:675-81.

4. Maini R, St Clair EW, Breedveld F, Furst D, Kalden J, Weisman M, et al. Infliximab (chimeric anti-tumour necrosis factor alpha monoclonal antibody) versus placebo in rheumatoid arthritis patients receiving concomitant methotrexate: a randomised phase III trial. ATTRACT Study Group. Lancet 1999;354:1932-9.

5. Lipsky PE, van der Heijde DM, St Clair EW, Furst DE, Breedveld FC, Kalden JR, et al; Anti-Tumor Necrosis Factor Trial in Rheumatoid Arthritis with Concomitant Therapy Study Group. Infliximab and methotrexate in the treatment of rheumatoid arthritis. Anti-Tumor Necrosis Factor Trial in Rheumatoid Arthritis with Concomitant Therapy Study Group. N Engl J Med 2000; 343:1594-602.

Personal non-commercial use only. The Journal of Rheumatology Copyright (C) 2015. All rights reserved 
6. van Vollenhoven RF. Switching between anti-tumour necrosis factors: trying to get a handle on a complex issue. Ann Rheum Dis 2007;66:849-51.

7. Cohen SB, Emery P, Greenwald MW, Dougados M, Furie RA, Genovese MC, et al; REFLEX Trial Group. Rituximab for rheumatoid arthritis refractory to anti-tumor necrosis factor therapy: Results of a multicenter, randomized, double-blind, placebo-controlled, phase III trial evaluating primary efficacy and safety at twenty-four weeks. Arthritis Rheum 2006;54:2793-806.

8. Keystone E, Emery P, Peterfy CG, Tak PP, Cohen S, Genovese MC, et al. Rituximab inhibits structural joint damage in patients with rheumatoid arthritis with an inadequate response to tumour necrosis factor inhibitor therapies. Ann Rheum Dis 2009;68:216-21.

9. Keystone EC, Cohen SB, Emery P, Kremer JM, Dougados M, Loveless JE, et al. Multiple courses of rituximab produce sustained clinical and radiographic efficacy and safety in patients with rheumatoid arthritis and an inadequate response to 1 or more tumor necrosis factor inhibitors: 5-year data from the REFLEX study. J Rheumatol 2012;39:2238-46.

10. Mease PJ, Cohen S, Gaylis NB, Chubick A, Kaell AT, Greenwald $\mathrm{M}$, et al. Efficacy and safety of retreatment in patients with rheumatoid arthritis with previous inadequate response to tumor necrosis factor inhibitors: results from the SUNRISE trial. J Rheumatol 2010;37:917-27.

11. Wolfe F, Michaud K. Towards an epidemiology of rheumatoid arthritis outcome with respect to treatment: randomized controlled trials overestimate treatment response and effectiveness. Rheumatology 2005;44 Suppl 4:iv18-iv22.

12. Greenberg JD, Kishimoto M, Strand V, Cohen SB, Olenginski TP, Harrington T, et al; Consortium of Rheumatology Researchers of North America Investigators. Tumor necrosis factor antagonist responsiveness in a United States rheumatoid arthritis cohort. Am J Med 2008;121:532-8.

13. Kievit W, Fransen J, Oerlemans AJ, Kuper HH, van der Laar MA, de Rooij DJ, et al. The efficacy of anti-TNF in rheumatoid arthritis, a comparison between randomised controlled trials and clinical practice. Ann Rheum Dis 2007;66:1473-8.

14. Zink A, Strangfeld A, Schneider M, Herzer P, Hierse F, Stoyanova-Scholz M, et al. Effectiveness of tumor necrosis factor inhibitors in rheumatoid arthritis in an observational cohort study: comparison of patients according to their eligibility for major randomized clinical trials. Arthritis Rheum 2006;54:3399-407.

15. Chatzidionysiou K, Lie E, Nasonov E, Lukina G, Hetland ML, Tarp $\mathrm{U}$, et al. Highest clinical effectiveness of rituximab in autoantibody-positive patients with rheumatoid arthritis and in those for whom no more than one previous TNF antagonist has failed: pooled data from 10 European registries. Ann Rheum Dis 2011;70:1575-80.

16. Chatzidionysiou K, Lie E, Nasonov E, Lukina G, Hetland ML, Tarp $\mathrm{U}$, et al. Effectiveness of disease-modifying antirheumatic drug co-therapy with methotrexate and leflunomide in rituximab-treated rheumatoid arthritis patients: results of a 1-year follow-up study from the CERERRA collaboration. Ann Rheum Dis 2012;71:374-7.

17. Soliman MM, Hyrich KL, Lunt M, Watson KD, Symmons DP, Ashcroft DM; British Society for Rheumatology Biologics Register. Effectiveness of rituximab in patients with rheumatoid arthritis: observational study from the British Society for Rheumatology Biologics Register. J Rheumatol 2012;39:240-6.

18. Soliman MM, Hyrich KL, Lunt M, Watson KD, Symmons DP, Ashcroft DM; British Society for Rheumatology Biologics Register. Rituximab or a second anti-tumor necrosis factor therapy for rheumatoid arthritis patients who have failed their first anti-tumor necrosis factor therapy? Comparative analysis from the British Society for Rheumatology Biologics Register. Arthritis Care Res 2012;64:1108-15.
19. Finckh A, Ciurea A, Brulhart L, Kyburz D, Möller B, Dehler S, et al; Physicians of the Swiss Clinical Quality Management Program for Rheumatoid Arthritis. B cell depletion may be more effective than switching to an alternative anti-tumor necrosis factor agent in rheumatoid arthritis patients with inadequate response to anti-tumor necrosis factor agents. Arthritis Rheum 2007;56:1417-23.

20. Finckh A, Ciurea A, Brulhart L, Moller B, Walker UA, Courvoisier $\mathrm{D}$, et al. Which subgroup of patients with rheumatoid arthritis benefits from switching to rituximab versus alternative anti-tumour necrosis factor (TNF) agents after previous failure of an anti-TNF agent? Ann Rheum Dis 2010;69:387-93.

21. Gottenberg JE, Ravaud P, Bardin T, Cacoub P, Cantagrel A, Combe $\mathrm{B}$, et al; AutoImmunity and Rituximab registry and French Society of Rheumatology. Risk factors for severe infections in patients with rheumatoid arthritis treated with rituximab in the autoimmunity and rituximab registry. Arthritis Rheum 2010;62:2625-32.

22. Kekow J, Mueller-Ladner U, Schulze-Koops H. Rituximab is more effective than second anti-TNF therapy in rheumatoid arthritis patients and previous TNF $\alpha$ blocker failure. Biologics 2012; 6:191-9.

23. Curtis JR, Jain A, Askling J, Bridges SL Jr, Carmona L, Dixon W, et al. A comparison of patient characteristics and outcomes in selected European and U.S. rheumatoid arthritis registries. Semin Arthritis Rheum 2010;40:2-14.e1.

24. Greenberg JD, Reed G, Decktor D, Harrold L, Furst D, Gibofsky A, et al; CORRONA Investigators. A comparative effectiveness study of adalimumab, etanercept and infliximab in biologically naive and switched rheumatoid arthritis patients: results from the US CORRONA registry. Ann Rheum Dis 2012;71:1134-42.

25. Sokka T, Kautiainen H, Toloza S, Makinen H, Verstappen SM, Lund Hetland M, et al; QUEST-RA Group. QUEST-RA: quantitative clinical assessment of patients with rheumatoid arthritis seen in standard rheumatology care in 15 countries. Ann Rheum Dis 2007;66:1491-6.

26. Kremer J. The CORRONA database. Ann Rheum Dis 2005;64 Suppl 4:iv37-41.

27. Kremer JM. The CORRONA database. Clin Exp Rheumatol 2005;23 Suppl 39:S172-7.

28. Karlsson JA, Kristensen LE, Kapetanovic MC, Gulfe A, Saxne T, Geborek P. Treatment response to a second or third TNF-inhibitor in RA: results from the South Swedish Arthritis Treatment Group Register. Rheumatology 2008;47:507-13.

29. Hyrich KL, Lunt M, Watson KD, Symmons DP, Silman AJ; British Society for Rheumatology Biologics Register. Outcomes after switching from one anti-tumor necrosis factor alpha agent to a second anti-tumor necrosis factor alpha agent in patients with rheumatoid arthritis: results from a large UK national cohort study. Arthritis Rheum 2007;56:13-20.

30. Grigor C, Capell H, Stirling A, McMahon AD, Lock P, Vallance R, et al. Effect of a treatment strategy of tight control for rheumatoid arthritis (the TICORA study): a single-blind randomised controlled trial. Lancet 2004;364:263-9.

31. Verstappen SM, Jacobs JW, van der Veen MJ, Heurkens AH, Schenk Y, ter Borg EJ, et al; Utrecht Rheumatoid Arthritis Cohort study group. Intensive treatment with methotrexate in early rheumatoid arthritis: aiming for remission. Computer Assisted Management in Early Rheumatoid Arthritis (CAMERA, an open-label strategy trial). Ann Rheum Dis 2007;66:1443-9.

32. Haraoui B, Pope J. Treatment of early rheumatoid arthritis: concepts in management. Semin Arthritis Rheum 2011;40:371-88.

33. Harrold L, Reed G, Magner R, Shewade A, John A, Greenberg J, et al. FRI0254 Comparative effectiveness of rituximab versus anti-tumor necrosis factor switching for rheumatoid arthritis patients. Ann Rheum Dis 2013;72 Suppl 3:A460. 
APPENDIX 1. Rituximab (RTX) initiators with 12-month followup and prior anti-TNF experience. For the purposes of our study, we excluded RTX initiations for the following reasons: (1) unclear date of RTX infusion ( $n=19)$, (2) no followup visits $(n=109)$, and (3) did not have a followup visit 12 months following RTX infusion $(n=111)$. This resulted in 376 RTX initiators with $>1$ year of followup. From this cohort, we identified a subset of patients $(n=265)$ who met the criteria for evaluation of response and adverse events. Specifically, from the 376 patients, we excluded those with no visit between RTX initiation and the 12-month followup visit $(\mathrm{n}=38)$, no assessment of disease activity using the CDAI at RTX initiation or at the 12-month followup visit $(\mathrm{n}=65)$, and initiation of RTX when in CDAI remission, which is seen when patients switch biologics for reasons such as cost or because of side effects to the prior agent $(\mathrm{n}=8)$. * CDAI score must have been measured within 6 months of initiation or after 2 months postinitiation. TNF: tumor necrosis factor; CDAI: Clinical Disease Activity Index.

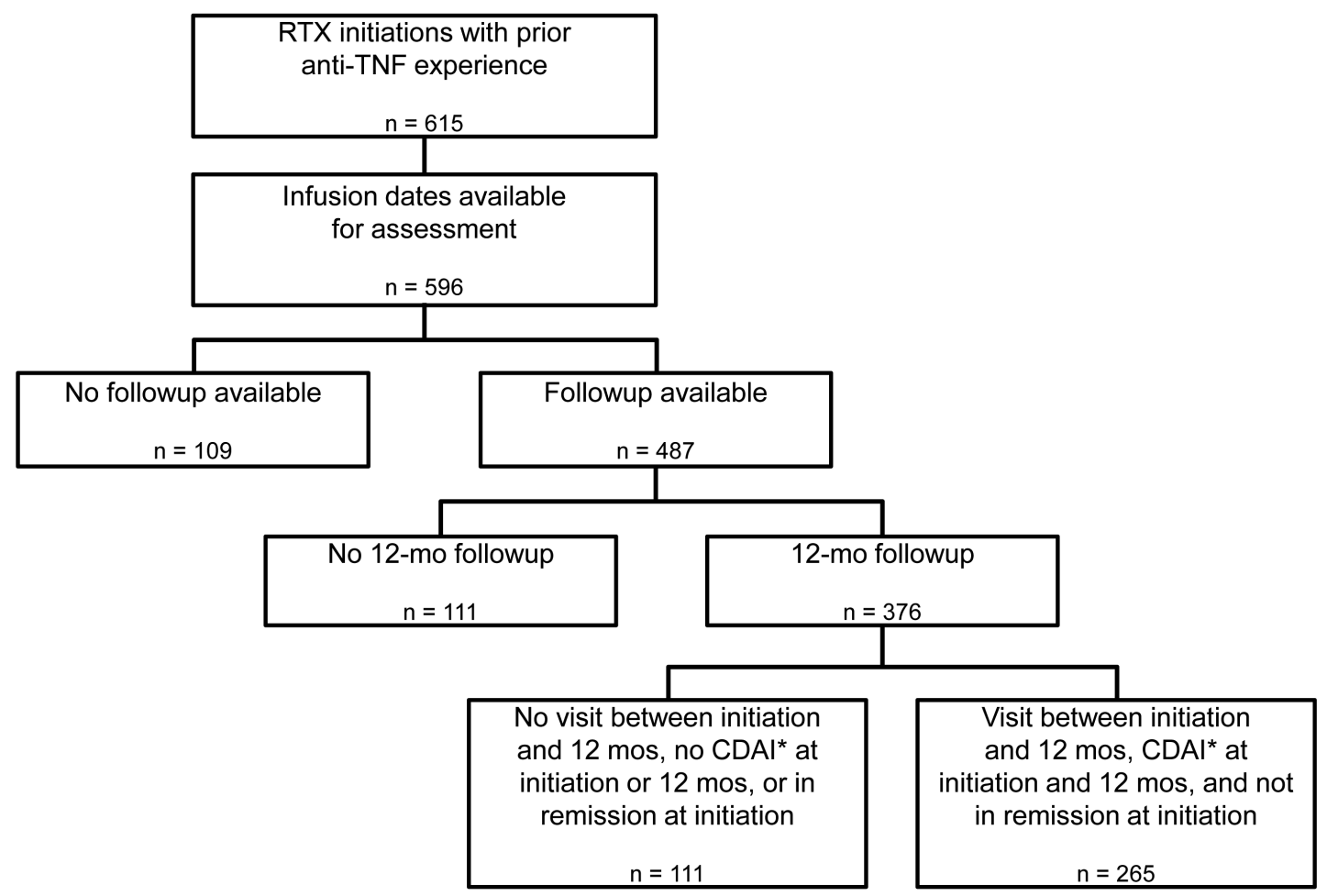

APPENDIX 2. Summary of rituximab (RTX) dosing and retreatment. Within CORRONA (COnsortium of Rheumatology Researchers Of North America), $>95 \%$ of all patients receiving RTX received the $2 \times 1000 \mathrm{mg}$ dose. Among those patients who received 1 prior anti-tumor necrosis factor (anti-TNF), 44 patients had 1 retreatment, 24 patients had 2 retreatments, and 6 patients had 3 retreatments. The mean (SD) time to retreatment for patients who received 1 prior anti-TNF was 7.1 (2.1) months. For those patients who received $\geq 2$ prior anti-TNF, 50 patients had 1 retreatment, 45 patients had 2 retreatments, and 9 patients had 3 retreatments. The mean (SD) time to retreatment for patients who received $\geq 2$ prior anti-TNF was 6.8 (1.8) months. 